\title{
Statistical estimation of oxygen atomic positions with sub Ångstrom precision from
} exit wave reconstruction

\author{
S. Bals, * S. Van Aert,* G. Van Tendeloo,* D. Van Dyck and D. Ávila-Brande ** \\ * EMAT-University of Antwerp, Groenenborgerlaan 171, Antwerp B-2020, Belgium
}

** Dpto. Química Inorgánica. Facultad de CC. Químicas. Universidad Computense de Madrid. Ciudad Universitaria s/n, 28040-Madrid. Spain

\begin{abstract}
Although high resolution transmission electron microscopy (HRTEM) is considered as the standard technique to study the atomic structure of materials, interpretation of HRTEM images is not always straightforward because of aberrations of the electromagnetic lens system. The information which can be obtained by qualitative HRTEM is therefore limited by the Scherzer point-resolution, but for a coherent electron source (field emission gun) the actual information limit of the microscope is beyond the Scherzer resolution. Using exit wave reconstruction, the information limit can be reached by correcting the phase changes due to the spherical aberration $\left(C_{s}\right)$ of the objective lens [1]. A great advantage of this technique is that the amplitude as well as the phase of the exit wave is reconstructed and since the light atom columns are revealed in the phase of the exit wave [2], it is possible to image oxygen atoms in the presence of heavier atoms.
\end{abstract}

We have applied exit wave reconstruction to determine an atomic model for the new compound $\mathrm{Bi}_{4} \mathrm{Mn}_{1 / 3} \mathrm{~W}_{2 / 3} \mathrm{O}_{8} \mathrm{Cl}$ of which the space group is determined to be $\mathrm{Cm} 2 \mathrm{~m}$ [3]. A focal series of 20 images with an equidistant focal decrease is recorded along the [1-10] zone axis and the numerical reconstruction of the exit wave is carried out using the TrueImage software [4]. After reconstruction and correction for the aberrations, the phase is extracted from the complex exit wave. As illustrated in Figure 1, the phase of the exit wave clearly shows the $\mathrm{Cl}$ atoms and the projected $\left\{\mathrm{WO}_{6}\right\} /\left\{\mathrm{MnO}_{6}\right\}$ octahedra with the oxygen atoms revealed as well. Two types of bismuth atoms can be distinguished in the image, i.e. $\mathrm{Bi}(1)$ and $\mathrm{Bi}(2)$. Around $\mathrm{Bi}(2)$, separate smaller dots corresponding to the oxygen atoms from the basal square plane can be observed, whereas we can observe the $\mathrm{Bi}(1)$ blobs with an extension corresponding to the oxygen atoms. Since the projected distance $\mathrm{Bi}(1)-\mathrm{O}$ is equal to $0.97 \AA$, which is close to the information limit, it is not possible to observe $\mathrm{Bi}(1)$ and $\mathrm{O}$ separately.

The phase of the exit wave is often considered as the final result, but in this study it is used as a starting point for quantitative refinement of the atom positions. This is done using statistical parameter estimation theory $[5,6]$. By adapting a parametric model to the experimental phase in the least-squares sense, it is possible to determine the atom positions. Using the Cramér-Rao Lower Bound as a measure of the precision, it can be shown that sub-Ångstrom precision is within reach. The result is presented in Figure 2, where the experimental phase is compared with the model evaluated at the estimated parameters [7].

References

[1] W.M.J. Coene et al., Ultramicroscopy 64 (1996) 109.

[2] D. Van Dyck et al., Ultramicroscopy 64 (1996) 1. 
[3] D. Ávila-Brande, work in progress

[4] Program TrueImage: Focal-series reconstruction package (Version 1.0.2, 2003, FEI company)

[5] A. van den Bos and A.J. den Dekker, Advances in Imaging and Electron Physics, 117 (2001) 241.

[6] A.J. den Dekker et al., submitted to Ultramicroscopy (2005)

[7] S. Bals and S. Van Aert are grateful to the Fund of Scientific Research-Flanders.

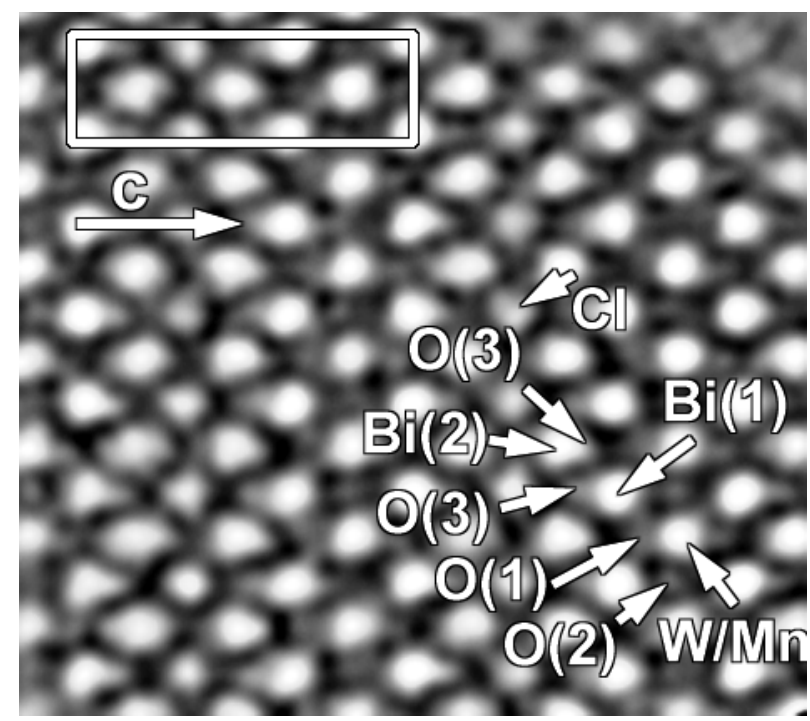

FIG. 1. Phase of the reconstructed exit wave along the [1-10] zone axis. The unit cell is outlined $(\mathrm{c}=14.1 \AA)$ and the positions of the different atoms are indicated.
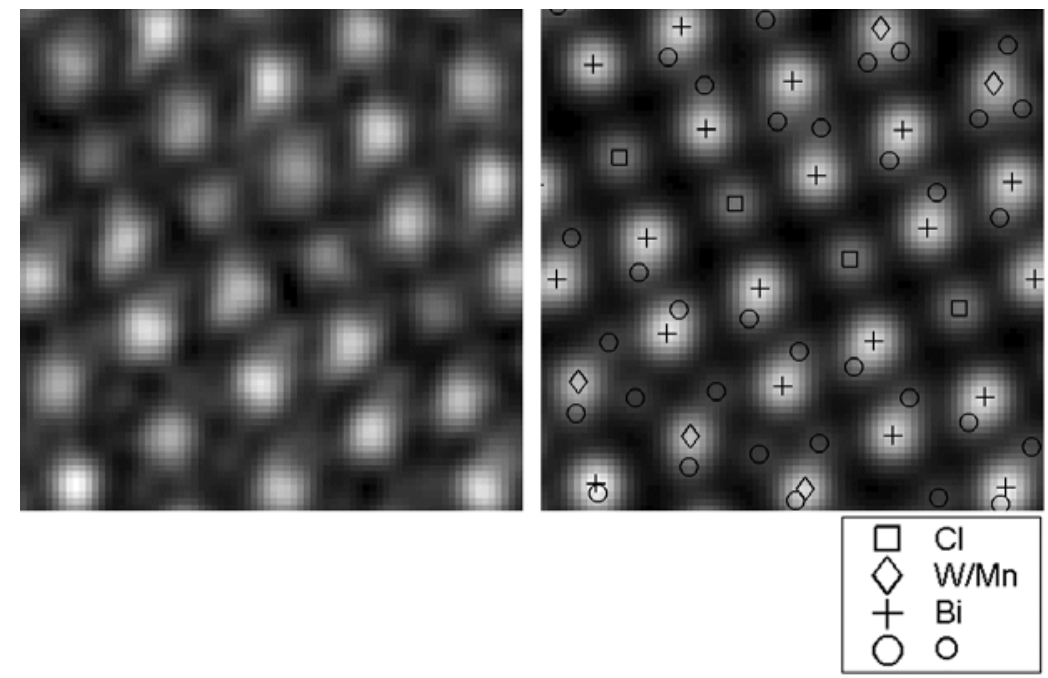

FIG. 2. The experimental phase of the exit wave is shown at the left of this picture, whereas the model evaluated at the estimated parameters is presented at the right. The atomic positions are indicated by the overlaying symbols. 